\title{
Importance of Pharmacoeconomics in the Pharmacovigilance for National Health Systems
}

\author{
Manuel Miguel Collazo Herrera ${ }^{1}$ and Leslie Pérez Ruiz ${ }^{2}$ \\ 1. National Institute of Hygiene, Epidemiology and Microbiology (INHEM), MINSAP (Ministry of Public Health of Cuba), \\ Havana 10 300, Cuba \\ 2. Center of Molecular Immunology (CIM), BioCubaFarma, Havana 11 600, Cuba
}

\begin{abstract}
The objective of this study is to conduct a literature review to assess the importance of pharmacoeconomics in developing pharmacovigilance activities as a working tool to guide the process of decision making in the health field. The authors have done a literature review in order to find information about pharmacovigilance and their relationship with the pharmacoeconomics field, as an important element to assess the economic and health consequences because of the use of drugs in health systems. From this study, it can be found that pharmacoeconomics applied to the pharmacovigilance activities should be considered as an aspect that contributes to improving the rational use of medicines, because it allows to compare the costs and consequences (beneficial and detrimental) with the use of different pharmacotherapeutic alternatives, in order to assess the negative effects due to ADRs (adverse drug reactions), which directly increase the morbidity and mortality in patients, increase the direct health costs and indirectly decrease the productivity labors. It can be also concluded that pharmacoeconomics constitutes an important aspect to the pharmacovigilance activity in the health systems, in order to evaluate the negative impact of ADR, both on patient health as its economic implications, due to associated costs with these adverse effects.
\end{abstract}

Key words: Pharmacoeconomics, pharmacovigilance, adverse drug reaction, health costs.

\section{Introduction}

The drugs have always been an important component of human life and social organizations, and their role is "essential to prevent diseases, protect and preserve health" $[1,2]$. In spite of drugs are not enough by themselves to provide adequate care, they play an important role in protection, maintenance and restoration of human health.

It should be noted that drugs are not only an essential component of health care and a symbol of treatment, they are also a commodity and an asset transaction, a product of modern industry; and also, they are an object of trade and sale [3]. Because of, the relationship between the drug as a marketing center and its use value is so decisive, the concept of "social good" has been originated in order to name

Corresponding author: Leslie Pérez Ruiz, M.Sc., research fields: pharmacology, pharmacy, pharmacoeconomics, cancer and neuroprotective drugs. these pharmaceutical products worldwide [2, 4].

Drugs have been a key factor in extending life expectancy, eradication or control of certain diseases and general welfare of the population. The importance of the use of drugs in the health of citizens requires that the employment of their benefit exceeds the risks that they can cause [5].

The benefit of a drug is expressed as the therapeutic effect shown in clinical trials and the risk that it may cause and its safety relating to the occurrence of adverse events, among other health implications. Therefore, risk-benefit ratio serves to express a judgment about the role of medicine in medical practice, based on data of its efficacy and safety, and in reference to their possible inadequate used [6].

For this reason, security is one of the main characteristics that should have a drug in order to be used with a very low probability to cause unjustifiable toxic effects, which could be an ADR (adverse drug 
reaction).

The objective of this paper is to describe the importance and need of the pharmacoeconomics for the development of pharmacovigilance role in health systems. For this purpose, it developed from a methodological point of view a literature review in order to find the necessary information on this topic at the international level. This research allows to do relevant analyzes to argue the interest in incorporating this tool, to valorize the negative consequences in terms of health and costs incurred due to ADR in the implementation of different health interventions, as an important element to decision making in the context of the NHS (National Health System).

\section{Adverse Drug Reaction: A Health Problem Worldwide}

ADR is an injury caused by taking a medication. It defined as any response to a drug which is noxious and unintended, including lack of efficacy (the condition that this definition only applies with the doses normally used) for the prophylaxis, diagnosis or therapy of disease, or for the correction or modification of physiological disorder function. Medication errors, such as overdose, and misuse and abuse of a drug, as well as drug exposure during pregnancy and breastfeeding, are also of interest, even without an adverse event, because they may result in an adverse drug reaction [1, 6-9]. ADRs may occur following a single dose or prolonged administration of a drug or result from the combination of two or more drugs. This term now includes the adverse clinical consequences of dependency, abuse and misuse of drugs and manifest as signs, symptoms, syndromes, abnormal lab tests and even diseases.

The study of ADRs is the concern of the field known as pharmacovigilance. An ADE (adverse drug event) refers to any injury occurring at the time a drug is used, whether or not it is identified as a cause of the injury. An ADR is a special type of ADE in which a causative relationship can be shown.When medicine is used in irrationally way, adverse reactions may occur due to its mechanism of action or pharmacological actions. ADRs are detected when the relevance of the indication, the treatment schedule, contraindications and potential drug interactions, among others $[10,11]$ is evaluated.

ADRs have a high frequency of occurrence and sometimes are considered serious adverse event when the patient outcome is one of the following: death, life-threatening, hospitalization (initial or prolonged), disability-significant, persistent, or permanent change, impairment, damage or disruption in the patient's body function/structure, physical activities or quality of life, congenital anomaly (birth defect) or requires intervention to prevent permanent impairment or damage [12].

ADRs are recognized as a common clinical-epidemiological problem in hospitals and emergency services, because they cause a negative impact on clinical outcomes of patients, increasing significantly the costs of hospital care and constitute a health problem worldwide [13, 14].

The magnitude of the effect caused by an ADR in an individual is related to its intensity or severity and should take into account its duration and the overall context in which this has occurred $[11,15]$. For that reason, the study of ADR and its evaluation can generate warning signals to health systems regarding the safety and use of medicines. These undesirable effects are a serious health problem worldwide, so their identification is an outstanding aspect in any health system.

All drugs are capable, in more or less frequency, of producing ADR. Some of them were detected during clinical trials. Others due to its low frequency of appearance require extensive population exposed to the drug by a longer time in order to detect undesirable effects [11-14, 16, 17]. Sometimes, the information about the safety of the drug is poor, so, in 
order to introduce it in the treatment of a particular disease, it must be subject to special surveillance. Therefore, the development of the pharmacovigilance in national health systems is necessary.

\section{Importance of Pharmacovigilance}

PV (pharmacovigilance), also known as drug safety, is the pharmacological science relating to the collection, detection, assessment, monitoring, and prevention of adverse effects with pharmaceutical products. Also, pharmacovigilance is a public health activity aimed at the identification, evaluation and prevention of risks associated with drugs once marketed. As such, it is geared to making decisions that will maintain the risk-benefit ratio of drugs in a favorable situation, or suspend use when this is not possible $[6,8,9,17]$

The WHO (World Health Organization) defines it as the science and activities relating to the detection, assessment, understanding and prevention of adverse effects or any other drug-related problem. The aims of $\mathrm{PV}$ are to enhance patient care and patient safety in relation to the use of medicines; and to support public health programmes by providing reliable, balanced information for the effective assessment of the risk-benefit profile of medicines [18-21].

Information received from patients and healthcare providers via PVAs (pharmacovigilance agreements), as well as other sources such as the medical literature, plays a critical role in providing the data necessary for pharmacovigilance to take place. In fact, in order to market or to test a pharmaceutical product in most countries, adverse event data received by the license holder (usually a pharmaceutical company) must be submitted to the local drug regulatory authority. Ultimately, pharmacovigilance is concerned with identifying the hazards associated with pharmaceutical products and with minimizing the risk of any harm that may come to patients. Companies must conduct a comprehensive drug safety and pharmacovigilance audit to assess their compliance with worldwide laws, regulations, and guidance.

Therefore, it can be considered pharmacovigilance as the set of activities designed to identify and evaluate the effects of acute and chronic use of pharmacological treatments in patients $[6,9,18,19]$. It includes the study of the beneficial and harmful effects, specifically the last one, because is known that any product with pharmacological activity may be a potential toxicant for human being.

Every day, pharmacovigilance systems face challenges due to the increasing number of molecules registered, the pressure from pharmaceutical companies on national regulatory agencies, the significant differences between clinical trials and common clinical practice, and the impossibility in many countries to do analytical studies in order to determine risk. Hence, the success of a pharmacovigilance system should converge on the political commitment of the health system; the academy to training of human resources capable to detect and analyze security issues, and adequate feedback about these important aspects in order to preserve the health of human beings by health professionals and general population.

A pharmacovigilance system should produce a useful visible response to their recipients, whom can encourage the individuals and institutions to prevent ARD and generate signals. Therefore, pharmacovigilance is an international task, based on the commitment and involvement of professionals as a way to contributing to patient safety through a scientific task of collective interest worldwide.

Among the main objectives of pharmacovigilance are found: identification of undescribed ARD, quantify the risk of adverse effects associated with drugs, prevent its occurrence and report on their detection, reporting, registration, monitoring, research and evaluation of the corresponding information [22-24]. In the environment of pharmacovigilance, the estimated costs because of treatment of ADR are a 
sensitive aspect in order to follow the safety of drugs on the market, as well as to assess the economic implications of these undesirable effects by performing pharmacoeconomic studies.

\section{Importance of Pharmacoeconomics for the Economic Valuation of ADR}

The indiscriminate use of drugs can produce adverse effects, in addition to the expected benefit. This leads to serious consequences for patients exposed and increases health care costs from the social/institutional point of view; and from the patient perspective. The evaluation of the drug must be based on a relative consideration of the cost, benefits and risks [2, 25].

ADRs as important causes of morbidity and mortality in the population, can be analyzed from the economic evaluation perspective, applying the methodology of description of costs and consequences from the health system perspective, because it compares all resources used in implementing the course of health action regarding to the consequences derived its use, with the objective of improving the process of decision making relating to the use of the drug [14, 26-30].

In the environment of pharmacovigilance, the estimating of costs because of ADRs treatment, especially avoidables is an important aspect to monitoring the safety of drugs on the market. It provides knowledge in their evaluation and it provides a strategy for prevention. Similarly, it should include economic evaluation research in the pharmacovigilance studies, time series, prognosis and other post-authorization studies, from the identification of drugs and pharmacological groups related to the occurrence of serious ADRs and avoidable severe ADRs [14, 26, 31].

Problems of safety, effectiveness and cost of ADRs are described in the international literature $[8,14]$ and adverse reactions have considerable importance in health systems and society, but according to the evidence demonstrated in published studies, are apply in specific hospital or region of a country. Besides that, the results differ among them because different reasons such as: the type of health system and the sources of information used. However, in spite of the recognized economic burden that ADRs have, the incorporating the economic analysis of these undesirable effects in the pharmacovigilance systems and health systems is still incipient [14, 31-33]. Drugs have an important economic impact for the NHS so it should be of concern to health administration. It is noteworthy that more than $20 \%$ of the health budget is spent on complications caused by drugs in the world $[14,26,31-36]$. For example, in the United States, annual costs associated with ADR are about \$2 billion $[37,38]$.

In Germany, a study published in 2011 resulted in that the health costs to treat ADRs was 816 million euros, the cost per patient was 381 euros, $58 \%$ of the costs was because hospitalizations, $11 \%$ due to consults and $21 \%$ because of treatment and long-term care [39]. In 2012, Spanish authors conducted a systematic review. In it, they concluded that studies about costs of ADRs have heterogeneous designs, assessed the direct health care costs at hospital and their results indicate that ADRs generate significant costs $[14,32]$.

Because of this situation, it is essential to consider pharmacoeconomics in order to do comparative analysis of the costs and consequences (both beneficial and detrimental) of the use of different pharmacotherapeutic alternatives, aimed to assess the impact of options or alternative actions on health patient. For this reason, the practical applications of pharmacoeconomics may be useful in situations relating to decision making, such as PMS (post-marketing surveillance) studies [26, 30].

In this regard, it is important to apply these assessments throughout the lifecycle of a health technology, because they generate information about its introduction and widespread use, in order to 
measure the impact of these interventions (both beneficial and harmful) in of routine clinical practice [40]. A negative impact on patient health, are the appearance of ADRs that increase morbidity and mortality. Economically, ADRs increase directly the cost of drug treatment, medical emergency consults and hospital admissions, and indirectly decrease the productivity labor because of lost working days.

\section{Conclusions}

From the study, we can find that pharmacoeconomics can be considered as a vital matter for the development of the pharmacovigilance, because it allows economically evaluation of ADRs and assess the negative impacts of these undesirable effects in the context of National Health System.

It is important to link both to be able to generate public health policies in a prospective way. This linkage is extremely important because, future ADRs can be monetized and budgeted within the NHS, generating significant savings. It is imperative that the Public Health Institutions have a Pharmacoeconomic Research Center, in order to know the additional expenditure that derives from the ADR. Only in this way will it be possible to carry out public health policies with sufficient support, which will bring important savings and also will anticipate future health problems, taking into account the aging of the population.

\section{References}

[1] Arias, T. 1999. Glossary of Medicines: Development, Evaluation and Use. Washington, D.C.: Organización Panamericana de la Salud.

[2] Collazo, M. 2004. "Pharmacoeconomics. Efficiency and Rational Use of Medicines." Brazilian Journal of Pharmaceutical Sciences 40 (4): 445-53.

[3] Velásquez, G. 2004. "Medications: Right or Merchandise?." Drugs Newsletter 7 (2): 4-7.

[4] Collazo, M., Ramos, D., Yañez, R., and De la Cruz, B. 2007. "Should Control of the Price of Medicines be a Responsibility of the National Regulatory Authorities?." Lat. Am. J. Pharm. 26 (6): 913-23.

[5] Goodman, L. S., and Gilman, A. 2012. The
Pharmacological Bases of Therapeutics. 12th ed. New York: Mc Graw Hill Interamericana.

[6] Jiménez, G., and Alfonso, I. 2010. "Risk Management in Pharmacovigilance." In Pharmacoepidemiology: Rational Use of Medicines, edited by Furones, J. A., Lara, C., Barbado, D. M., Jiménez, G., Pérez, J., and Cruz, M. A. Havana: Academy Editorial, 120-42.

[7] Urmimala, S., López, A., Maselli, J., and González, R. 2011. "Adverse Drug Events in U.S. Adult Ambulatory Medical Care." Health Services Research 46 (5): 1517-33.

[8] Lazarou, J. 1998. "Incidence of Adverse Drug Reactions in Hospitalised Patients: A Meta-analysis of Prospective Studies." JAMA 279: 1200-5.

[9] Pharmacovigilance Group. 2011. Pan American Network for Pharmaceutical Harmonization. Good Pharmacovigilance Practices for the Americas. 5th ed. Washington, D.C.: Pan American Health Organization.

[10] WHO (World Health Organization). 2005. "Drug Safety. Adverse Drug Reactions. Fact Sheet No. 293." WHO. Accessed November 23, 2006. http://www.who.int/media centre/factsheets/fs293/es/print.html.

[11] Ramírez, R. C., Faúndez, L. E., and Orellana, B. R. 2011. "Report of Adverse Reactions to Medicinal Products with Mucocutaneous Involvement in Clinical Hospital Universidad de Chile during the Years 2004-2010.” Chile Clinical University Hospital Journal 22: 104-12.

[12] Passarelli, M. C., Jacob-Filho, W., and Figueras, A. 2005. "Adverse Drug Reactions in an Elderly Hospitalised Population: Inappropriate Prescription Is a Leading Cause." Drugs Aging 22 (9): 767-77.

[13] Salas, S. G., Pérez, M. E., Meléndez, S. G., and Castro, L. I. 2012. "Adverse Drug Related Hospital Admissions and Stays Reactions: Systematic Review 2000-2011." Mexican Journal of Pharmaceutical Sciences 43 (3): 19-35.

[14] Vallano, A., Escasan, A., Pedrós, C., and Arnau, J. M. 2012. "Systematic Review of Studies Assessing the Cost of Adverse Drug Reactions." Gac Sanit 26 (3): 277-83.

[15] Mira, J., Cho, M., Montserrat, D., Rodríguez, J., and Santacruz, J. 2013. "Key Elements in the Implementation of Reporting Systems of Hospital Adverse Events in Latin America." PAHO 33 (1): 1-7.

[16] Pouyanne, P., Haramburu, F., Imbs, L., and Beagud, B. 2000. "Admission to Hospital Caused by Adverse Drug Reactions: Cross Sectional Incidence Study.” BMJ 320: 1036.

[17] Vries, E. N., Ramrathan, M. A., Smorenburg, S. M., Gouma, D. J., and Boermeester, M. A. 2008. "The Incidence and Nature of In-hospital Adverse Events: A Systematic Review." AMQualSaf Health Care 17: 216-23. 
[18] Chao, A., Pérez, J., and Debesa, F. 2010. "Pharmacovigilance." In Pharmacoepidemiology: Rational Use of Medicines, edited by Furones, J. A., Lara, C., Barbado, D. M., Jiménez, G., Pérez, J., and Cruz, M. A. Havana: Academy Editorial, 1-96.

[19] Alfonso, I., and Jiménez, G. 2010. "Pharmacovigilance Methods." In Pharmacoepidemiology: Rational Use of Medicines, edited by Furones, J. A., Lara, C., Barbado, D. M., Jiménez, G., Pérez, J., and Cruz, M. A. Havana: Academy Editorial, 98-119.

[20] WHO (World Health Organization). 2001. "Monitoring Drug Safety. Guide for Installation and Operation of a Pharmacovigilance Centre." The Uppsala Monitoring Centre. Accessed November 15, 2010. http//www.who-umc.org.

[21] Uppsala Monitoring Centre. 2012. "Uppsala Report." Uppsala Monitoring Centre. Accessed February 8, 2013. http//www.who-umc.org.

[22] Ponte, L. M., Ragusa, M., Armenteros, A., and Wachs, A. 2013. "Relevance of Hospital Pharmacovigilance in Current Medical Practice." Medical (B. Aires) 73 (1): 35-8.

[23] Hernández, M., and Juárez, H. 2010. "Pharmacovigilance in Pediatrics." Acta Pediatr Mex. 31 (5): 227-32.

[24] Sánchez, I., Amador, C., Plaza, J. C., Correa, G., and Amador, R. 2014. "Clinical Impact of Active Pharmacovigilance System by a Pharmacist in Reporting and Under-Reporting of Adverse Drug Reactions." Medical Journal of Chile 142: 998-1005.

[25] Collazo, M., and Sosa, I. 2011. "Pharmacoeconomics Should It Be of Interest to Evaluate the Efficiency in Decision-Making?." Colombian Journal of Pharmaceutical Sciences 40 (1): 54-66.

[26] Iñesta, G. A. 2011. Drug and Pharmacoeconomics. Madrid: National School of Health \& Health Institute Carlos III.

[27] Carswell, C. I., and Paladino, J. A. 2005. "Reporting Pharmacoeconomic Evaluations." Pharmacoeconomics 23 (11): 1973-4.

[28] Mason, J. M., and Mason, A. R. 2006. "The Generalisability of Pharmacoeconomic Studies: Issues and Challenges Ahead." Pharmacoeconomics 24 (10): 937-45.

[29] Drummond, M. F., Sculpher, M. J., Torrance, G. W., O'Brien, B. J., and Stoddart, G. L. 2005. Methods for the Economic Evaluation of Health Care Programmes. 3th ed. Cary: Oxford University Press.

[30] Waley, T., Hayco, A., and Boland, A. 2005. Pharmacoeconomics. Madrid: Elsevier Spain. (in Spanish)

[31] Segura, O., and Maldonado, C. 2003. "Adverse Drug Reactions: An Approach from an Economic Point of View." Biomedical 23: 401-7.

[32] Allué, N., Chiarello, P., Bernal, D. E., Castells, X., Giraldo, P., Martínez, N., etc. 2014. "Economic Impact of Adverse Events in Spanish Hospitals from the Basic Minimum Data Set.” Gac Sanit 28 (1): 48-54.

[33] Pinzón, F., and Maldonado, C. 2011. "Direct Costs and Impact on Hospital Morbidity and Mortality from Preventable Adverse Drug Events in a Third Level Institution Bogota." Biomedical (Bogotá) 31 (3): 307-15.

[34] Apretna, E., Haismburn, F., Taboulet, F., and Begand, B. 2005. "Medical and Socio-economical Impact of Drug-Induced Adverse Reactions." Press Med. 34 (4): 271-6.

[35] Lagnaoui, R., Moore, N., Fach, J., Longy-Boursier, M., and Bégaud, B. 2000. "Adverse Drug Reactions in a Department of Systemic Diseases-Oriented Internal Medicine: Prevalence, Incidence, Direct Cost and Avoidability." Eur. J. Clin. Pharmacol. 55: 181-6.

[36] Collazo, M. 2015. "Pharmacoeconomics: Key Issue for the Development of Pharmacovigilance." VITAE Journal 22 (Supl 1): S22-S23.

[37] Gyllensten, H., Rehnberg, C., Jönsson, A. K., Petzold, M., Carlsten, A., and Sundel, K. A. 2013. "Cost of Illness of Patient-Reported Adverse Drug Events: A Population-Based Cross-Sectional Survey.” BMJ Open. Accessed April 28, 2014. http://dx.doi.org/10.1136/ bmjopen-2013-002574 e002574.

[38] Lagnaoui, R., Moore, N., Fach, J., Longy-Boursier, M., and Bégaud, B. 2000. "Adverse Drug Reactions in a Department of Systemic Diseases-Oriented Internal Medicine: Prevalence, Incidence, Direct Cost and Avoidability." Eur. J. Clin. Pharmacol. 55: 181-6.

[39] Stark, R. G., John, J., and Leid, R. 2011. "Health Care Use and Costs of Adverse Drug Events Emerging from Outpatient Treatment in Germany: A Modelling Approach." Starketal BMC Health Services Research 11: 1-9

[40] Martínez, J. 2006. "Health Technology Assessment: Quality Assurance and Efficiency of the NHS." Spanish Health Economics Journal 5 (6): 312-3. 